\title{
Force Control of a Scara Robot using Neural Networks
}

\author{
Fernando Passold \\ Dept. of Electrical Engineering \\ University of Passo Fundo \\ Passo Fundo, Brazil \\ passold@upf.tche.br
}

\begin{abstract}
This paper describes experimental results achieved applying artificial neural networks (NNs) trying to perform force/position control of a real scara manipulator robot. The general control strategy consists of a neural controller that operates in parallel with a conventional controller based on feedback error learning architecture. There are used two NNs: one for the position loop control and another to the force loop control based on the structure knew as hybrid force/position control. The main purpose was lets the NNs compensates dynamical effects that arises when a manipulator is in contact with an environment. Successfully results have achieved for the position loop control but practical problems was observed related to force loop control using NNs even with different sets of input data for this NN. Practical problems are discussed.
\end{abstract}

\section{Introduction}

This paper describes and discusses practical results obtained with the use of computational intelligence techniques, specifically artificial neural networks, applied to a complex control problem: the force/position control of a real manipulator robot.

This work was applied in a real scara robot installed at the Laboratory of Industrial Automation of the Federal University of Santa Catarina. The work is the result of cooperation between the Automation and Mechanical Engineering departments. The robot was manufactured by the Institute of Robotics (IfR) of the ETH (Swiss Federal Institute of Technology, http://www.ifr.mavt.ethz.ch/). Differently from most industrial manipulator robots, this one comes with an open architecture, which allows the freely implementation of any type of control law. The main purpose was to evaluate new algorithms for force/position control, since this robot is also equipped with a force sensor. Figure 1 shows the INTER scara robot.

\author{
Marcelo Ricardo Stemmer \\ Dept. of Automation System \\ Federal University of Santa Catarina \\ Florianópolis, Brazil \\ marcelo@das.ufsc.br
}

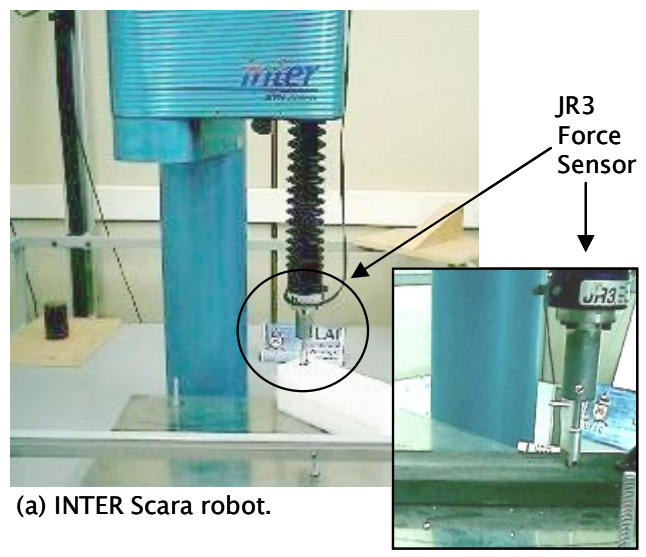

(b) Robot performing force control.

Figure 1. INTER Scara Robot.

The mainly application of manipulator robots are with tasks that do not evolve contact with the surrounding environment like in operations as arc and spot welding, spray painting, laser and water jet cutting [10]. Even pick-and-place operations only require position control. And some industrial applications as assembly mechanical groups could be solved using position control techniques in some cases based on impedance position control techniques since not precise force control are required, only a little pressure (pushing). But for task as milling, drilling, deburring and grinding, force control is desirable since many manipulators are not backdriveable because of their gearing, then reaction forces will cause damage to the manipulator [11]. Even very small position errors could result in large reaction forces exerted on the arm [11]. One simplistic solution it to insert mechanical compliance between the position controlled manipulator and the environment. However, this compliance is open loop, and adds uncontrolled degrees of freedom to the manipulator structure [11].

A new kind of problem arises when one wants to move robot to specific locations in its environment and controls impart specific forces as in polishing task, a labor intensive field that requires skilled machinists. The problems becomes since imprecise location of the mechanical part to be machined, imprecise description of the environment, detailed path trajectories becomes 
difficult to obtain due the complex trajectory required in tasks operations as polishing, and even unpredictable surface finish arose due to machining variations including differences from tool changes and spindle vibrations [6][10]. So, this categorizes a non-linear and time variant system where controllers with adaptation capabilities to deal with this kind of difficulties are desirable.

Zeng and Hemami [12] have reported some traditional approaches used to establishes force control, since implicit to explicit methods (respectively without and with an force sensor). Basically implicit approaches try to estimate the contact force $(h)$ in a indirect way based on the estimative of the environment stiffnes matrix $\mathrm{K}$ and the displacement $(d x)$ carry out inside the environment $(h=K \cdot d x)$. This is a common technique employed when a expensive force sensor is not available. Even impedance force control approach could be done in this manner and is used for simple force control tasks. The fundamental philosophy of impedance control is that the manipulator control system should be designed not to track a motion trajectory alone, but rather to regulate the mechanical impedance of the system manipulator-environment [12]. Impedance control requires commanded position based on position and force measurements done.

Since force control of the manipulator becomes necessary at least one of the degrees of freedom (d.o.f.) of the manipulador; the other d.o.f. remain position controlled, another simply approach arise called hybrid control [11][10]. Simply put, the manipulator should be controlled in directions in which the position is constrained by environmental interaction, and position controlled in all orthogonal directions. The Hybrid Control formalism does not specify what particular type of force control should be used. It only partitions the space spanned by the total d.o.f. into one subspace in which position control is employed, and another in which the force control is employed.

Another last challenge is how to balance position and force controls as usually only pure force control are not required.

\section{Neural Controller Types}

Artificial neural network (ANN) are biologically inspired on real neural networks which exhibit a very powerful mechanism to deal with a massive number of data elements in a non-linear and complex way and beyond these facts, it also owns the ability of learning. ANNs have been applied to several cases of control systems, showing special adequacy when we have to deal with complexity, non-linearity or uncertainty [9]. The neural approach is interesting notably in the cases where: a) Mathematical models of the process are poor or do not even exist and linear models are inadequate to represent the system with sufficient accuracy;

b) The system works satisfactorily with an existing controller but its performance deteriorates substantially when high performances (e.g. speeds) are required, so non-linear controllers become necessary.

ANNs have proved their ability to approximate arbitrary nonlinear maps and the availability of methods for adjusting their parameters on basis of input and output data makes them particularly attractive when unknown nonlinearities are present in a system [2][1]. They could be used to: map non-linear functions; perform operations between multiple inputs / output variables (exceeding the correlation capability of traditional statistic methods); become a part of an adaptive control schema (in this case, the nets are trained on-line); or to identify systems in real-time. In the control systems area, a few neural models have been proved to be more suitable than others, namely the:

1) Multilayer Perceptron networks (MLP);

2) Radial Base Function networks (RBF).

Katic and Vukobratovic [3], Morris and Khamanissia [8] underlie two learning architectures that seems to be the most appropriate and promising: a) Feedback-error learning architecture; b) Adaptive learning architecture. In this work it was explored the feedback-error learning architecture. The feedback error learning approach is characterized by the ANN inserted in the feedback path to capture the nonlinear characteristics of the system The ANN weights area tuned on-line with no off-line learning phase and, when compared with the adaptive technique, we do not require any knowledge of the robot dynamics, linearity in the unknown system parameters or the tedious computation of a regression matrix. Thus, this approach is model-free and represents an improvement over adaptive techniques [5]. Narendra [9] also comments that it seems to be valuable to keep linear and non-linear components working in parallel, where the neural networks represent the non-linear components. He also mentions the brief learning time periods and the increase of accuracy that results from this kind of integration.

\section{Hybrid Integrate Controller Proposed}

It was used ANNs performing in parallel with a PD/PID controller inside the hybrid controller structure since its formalism does not specify what particular type of position or force control should be used. So, the manipulator was force controlled in directions constrained by the environment interaction and position controlled in the others orthogonal directions. 


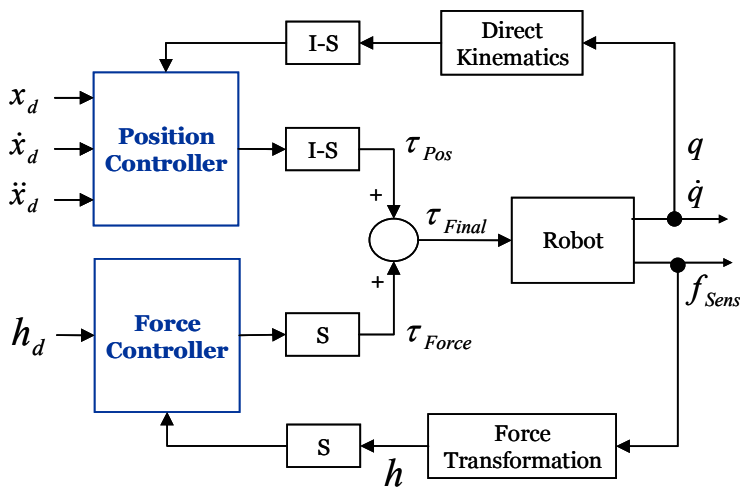

Figure 1. Block diagram of hybrid control.

Each loop of control use its own integrate controller, that means: for the position control loop was used a PID performing in parallel with an MLP or RBF ANN, and for the force control loop it was used a PI performing in parallel with another MLP or RBF ANN.

\subsection{Integrate position controller}

Figure 2 shows the integrate position controller used.

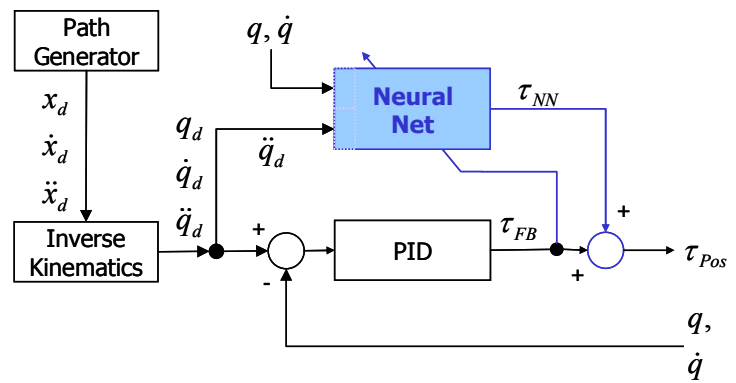

Figure 2. Integrate position controller.

Both MLP and RBF uses the same input vector data: $x_{N N}=\left[\begin{array}{lll}q & \dot{q} & \ddot{q}_{d}\end{array}\right]$, where $q$ is the vector of actual join positions; $\dot{q}$ is the vector of actual join velocities (obtained through numerical differentiation); $\ddot{q}_{d}$ corresponds to the desired join accelerations (like in other manipulators, there is no accelerometer available for each join, so the desired acceleration computed by the path generator was used). Each vector is of dimension 4 corresponding to the 4 d.o.f. of the scara robot used.

The MLP net used consists of 4 layers (2 hidden) with 12 $\times 8 \times 5 \times 4$ neurons. These inputs were bounded into its maximum and minimum possible operational values for this robot and then scaled between the neural input range -0.9 to +0.9 only for the MLP nets. And the same was done related to the output torque computed by the both ANNs used.
However, different from MLP nets, the RBF nets does not need a scale procedure as it could deal directly with rough data, but it was necessary to organize the input data into three different classes: 1) join positions, 2) join velocities and 3) join accelerations. The data related to each joint was divided into these three classes and each one mapped using $m$ Gaussian functions, so for the first joint we have to map 3 different kind of data: $q_{0}$ (angular position), $\dot{q}_{0}$ (angular velocity, numerically obtained) and $\ddot{q}_{0 d}$ (desired joint acceleration) and so was done to the other 3 joints. All these neurons of the hidden layer have been connected to the neurons of the output layer.

The RBF net was implemented using 5 Gaussian Function for each element of the input vector data:

$$
\mathbf{G}\left(\mathbf{x}, \mathbf{x}_{i}\right)=\exp \left(-\frac{1}{2 \sigma_{i}^{2}}\left\|\mathbf{x}-\mathbf{x}_{i}\right\|^{2}\right)
$$

where $\mathbf{G}(\cdot, \cdot)$ represents the vector of Gaussian functions; $\mathbf{x}$ is related to the input vector data; $\mathbf{x}_{i}$ correspond to the $i$ th center of the Gaussian function; $\sigma$ represents the spread (or standard deviation) of the Gaussian functions; $i$ varies between 1 and $m$ Gaussian functions used to map the iput each input data. That results in a net with an input (passive) layer using 12 neurons, an hidden layer (responsible of the non-linear transformation from input vector space to the internal vector space) with 3 classes $\times$ 4 d.o.f. $\times 5$ Gaussian functions) $=60$ neurons finishing with an output layer of 4 neurons each one related to the output torque required for each joint of the scara robot. The centers $\mathbf{x}_{i}$ of the $m$ desired Gaussian functions was uniformly distributed, based on the range (minimal and maximal values) of the input vector and so was done to also fix the width (or standard deviation) of the $m$ Gaussian Functions used (see [1] and [2] for further details).

The synaptic weights of the MLP net and the output synaptic weights of the RBF net were adjusted on-line using the traditional back propagation algorithm expanded with momentum term $(\alpha)$. The error signal used was the output torque computed by the conventional controller. The momentum term was introduced to accelerate the decent steepest back propagation algorithm as also prevent the net to get paralyzed into a local minimum of its error surface [2].

The equation of the PID used to perform in parallel with the ANNs is given by:

$$
\boldsymbol{\tau}_{F B}=\mathbf{B}(\mathbf{q}) \cdot\left(\mathbf{K}_{p} \widetilde{\mathbf{q}}+\mathbf{K}_{i} \int \widetilde{\mathbf{q}} d t+\mathbf{K}_{d} \dot{\widetilde{\mathbf{q}}}\right)
$$

where $\boldsymbol{B}(\boldsymbol{q})$ refers to the inertia matrix of the robot (estimated); $\widetilde{\mathbf{q}}=\left(\mathbf{q}_{d}-\mathbf{q}\right)$ represents the error between the desired and the actual joint position; $\dot{\widetilde{\mathbf{q}}}$ refers to the velocity error; $\mathbf{K}_{p}$ is the vector of proportional gains for 
each joint; $\mathbf{K}_{i}$ refers to the integral vector gains and $\mathbf{K}_{d}$ is the derivative gain vector for each join. To get a PD action over the system, the $\mathbf{K}_{i}$ vector was not used (equal to zero). Table 1 shows the parameters used for the tested PID controllers.

Table 1. PID parameters used (position loop).

\begin{tabular}{|r|r|r|r|r|}
\hline & Joint 0 & Joint 1 & Joint 2 & Joint 3 \\
\hline \hline $\mathrm{Kp}$ & 4900 & 12100 & 90000 & 14400 \\
\hline $\mathrm{Kd}$ & 140 & 220 & 600 & 240 \\
\hline $\mathrm{Ki}$ & 478 & 1200 & 9200 & 1410 \\
\hline
\end{tabular}

\subsection{Integrate force controllers}

The conventional force controller used in the force control loop was a PI defined as:

$$
\tau_{F}=K p \cdot \tilde{h}+K_{i} \int \tilde{h} d t
$$

where: $K_{p}$ and $K_{i}$ correspond to proportional and integral gains of the PI controller; $\tilde{h}=h-h_{d}$, correspond to the "force error". The integral action was proved necessary to guarantee null final error since only a proportional controller could not compensate friction effects evolved to do little movements with the joints (large $K_{p}$ gains quickly results in instabilities to a system in contact with the environment).

There were tested 4 structures of neural nets as the first one (RNf-1) have achieved low performance. They vary on the input vector used:

$$
\begin{aligned}
& \text { 1. RNf-1: } x_{N N}=\left[\begin{array}{lll}
q & f_{\text {Sens }} & h_{d}
\end{array}\right] ; \\
& \text { 2. RNf-2: } x_{N N}=\left[\begin{array}{llll}
h_{z} & \tilde{h}_{z}
\end{array}\right] ; \\
& \text { 3. RNf-3: } x_{N N}=\left[\begin{array}{lllll}
h_{z} & h_{z} z^{-1} & \ldots & h_{z} z^{-5} & h_{d_{z}}
\end{array}\right] ; \\
& \text { 4. RNf-4: } x_{N N}=\left[\begin{array}{lll}
h_{z} & \tilde{h}_{z} & \dot{q}_{2}
\end{array}\right],
\end{aligned}
$$

where $q$ is the angular position vector; $f_{\text {Sens }}$ correspond to the output data vector of force sensor (it is of dimension 6 as there are forces and moments information related to each XYZ axis of the force sensor); $h_{d}$ corresponds to the vector of measured forces already converted form sensor force space to the base coordinates of the robot: $h=N\left(f_{\text {Sens }}, q\right) ; h_{z}$ is the measured force over $\mathrm{Z}$ axis already expressed over the operational space (base coordinates of the robot); $\tilde{h}_{z}$ represents the error force over the $\mathrm{Z}$ axis of the robot; $h_{z} z^{-n}$ correspond to the $n$-th delayed information about forces measured over the $Z$ axis; and $\dot{q}_{2}$ is the linear velocity measured of the prismatic joint of the scara robot (responsible of the movements over the $\mathrm{Z}$ axis).
The first net (RNf-1) was the first net tested for force control and thus it haves 4 outputs, each one related to the each joint of the robot. In directions that it was not desirable force interaction the elements of vector $h_{d}$ must be defined as zero. The MLP tested use 4 layers containing $14 \times 10 \times 6 \times 4$ neurons. As good performance could not be reached the remained neural force nets were tested only to control force in $\mathrm{Z}$ direction using MLP ANNs (slower than RBF nets).

\section{Experimental Results}

The proposed controllers were tested executing a positional control over the XY plane with final orientation fixed and force control specified only in $\mathrm{Z}$ direction. The robot moves over a line near $34(\mathrm{~cm})$ of length at $5(\mathrm{~cm} / \mathrm{s})$ exercising force against an elastic surface maintaining a force contact $h_{d_{z}}=5(\mathrm{~N}) \cong 0.5(\mathrm{Kg})$ - see Fig 1(b) and Fig. 4.

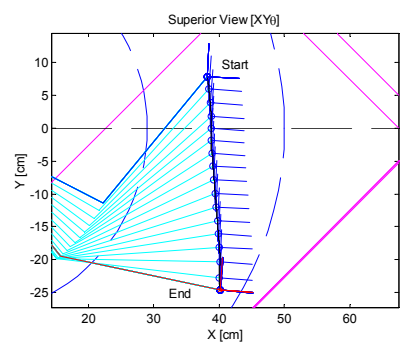

(a) Superior view

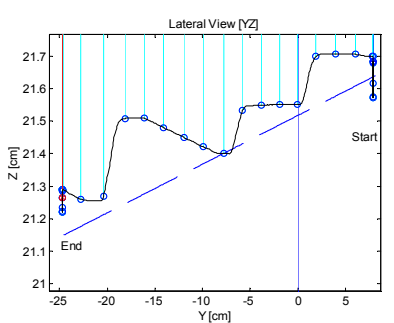

(b) Lateral view.
Figure 4. Movement of the robot.

The PID+RBF position controller performs very successfully as could be seen in figure 5 .

Figure 6 shows the results achieved with the RNf-1 force controller where the PI performs with $K_{p}=200$ and $K_{i}=20$ and the $\mathrm{NN}$ with $\eta=0.035$ (learning rate) and $\alpha=0.5$ (momentum term). It could be observed that the ANN only contributes with non null torque in the beginning of the trajectory before the middle point of the trajectory. The PI force controller working alone achieves better performance: $\operatorname{Max}\left\{h_{d}\right\}=10.87(\mathrm{~N}), \bar{h}_{d}=5.15 \pm 2.53(\mathrm{~N})$.

Increasing the learning rate until $\eta=0.035$ only results in more oscillations in force control loop with lost of contact with the surface (in this case, the control algorithm implemented automatically changes to pure position control and the contact could be recovery). The first trials have made with the force input vector of the ANN operating with the full scale of the force sensor: $\operatorname{Max}\left\{f_{\text {Sens } Z}\right\}=200(\mathrm{~N})$. Before this, these input elements have their range reduced off $25 \%$ but better results could not been achieved. Test with the RNf-1 operating with an RBF net results in torques spikes over than the $877(\mathrm{Nm})$ supported by the motor of joint 2 (the prismatic one) in 


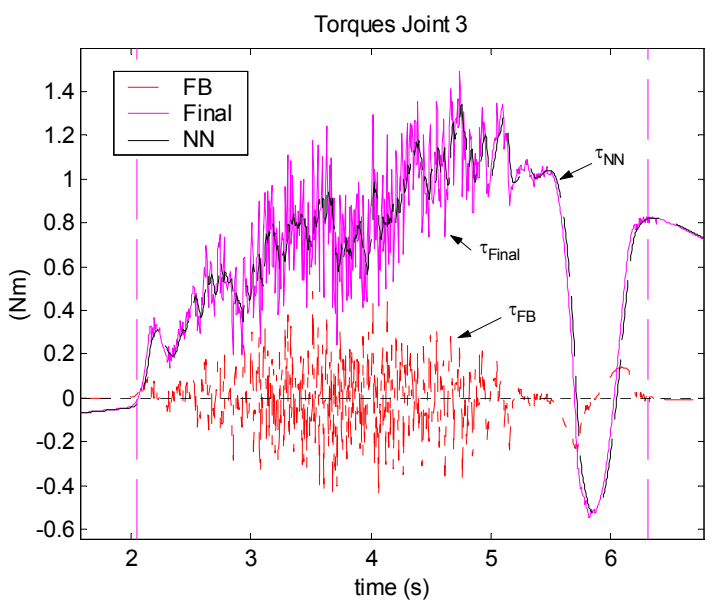

Figure 5. Output torque developed for joint 3 by the PID+RBF position controller.
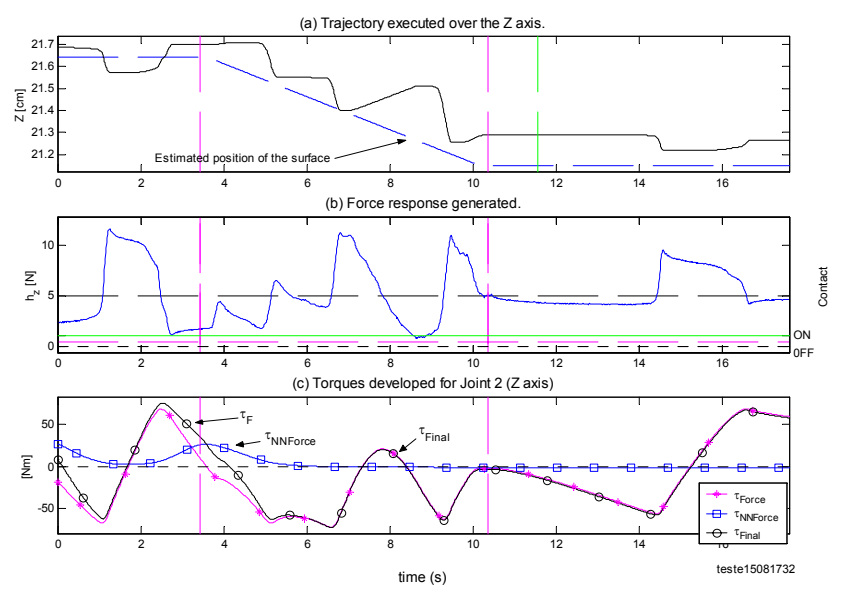

Figure 6. Results achieved by RNf-1.

less than 0,138 seconds (corresponding to 69 sampling times only) - emergency security functions acts in these cases stopping the robot to prevent damages (there was also detected $\left.f_{z}>80(\mathrm{~N})\right)$. These net was operating with $\eta=0.005$ and $\alpha=0.5$. The faster performance of $\mathrm{RBF}$ compared to the MLPs was already realized with the integrate position controller when the RBF best operates with $\eta=0.005$ and $\alpha=0.5$. These net was also tested before and after the re-scaling procedure for the elements related to the force sensor measurements and thus the problem is not related to the center positions of the Gaussian functions (adaptive learning for this parameter possible will not lead to better results).

So the RNf- 2 was tested using a MLP with only $2 \times 3 \times 1$ neurons with $\eta=0.001$ and $\alpha=0.5$ - their results are showed in figure 7 .

It could be seen that better results are achieved notably during the return to the start position with $\bar{h}_{d}=7.822 \pm 6.34(\mathrm{~N})$ but not as good as a PI alone.
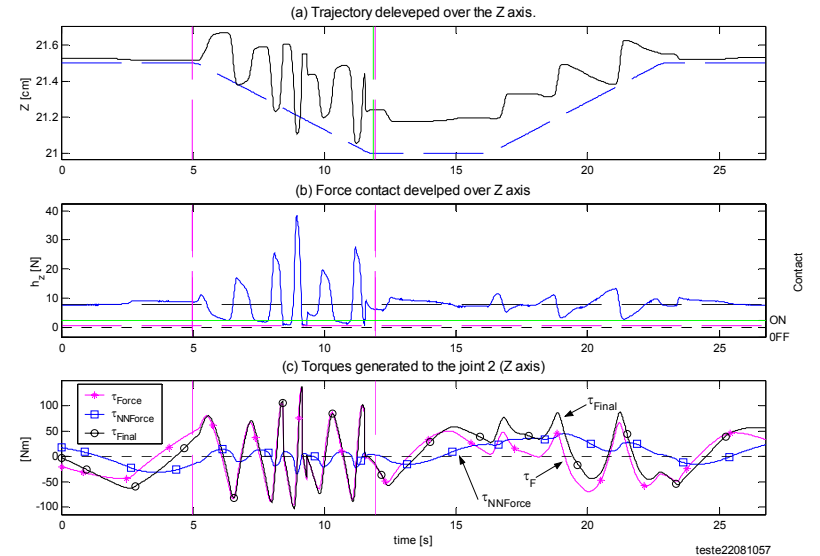

Figure 7. Results achieved by RNf-2.

Figure 8 shows the results achieved with the RNf-3, a MLP net with $2 \times 3 \times 1$ neurons, $\eta=0.035$ (smaller values paralyzes the net) and $\alpha=0.5$, but now the desired force contact was increased to $h_{d_{z}}=1.0(\mathrm{~N})$. As showed by figure 8 this net with its set of data was achieved the best results but only when the robot was moving over the surface. When it stops, the force loop control enter into an oscillatory state doing someone think if the velocity developed over the directions with constraints could be a relevant data for the force loop controller.

So finally figure 9 shows the results obtained with the RNf-4 net implemented with a MLP net with $3 \times 4 \times 1$ neurons, $\eta=0.02$ and $\alpha=0.5$. It can be noted that the inclusion of the velocity with which the robot enters the environment, $\dot{q}_{2}$, does not worth. These net quite does not contribute to the force loop control.
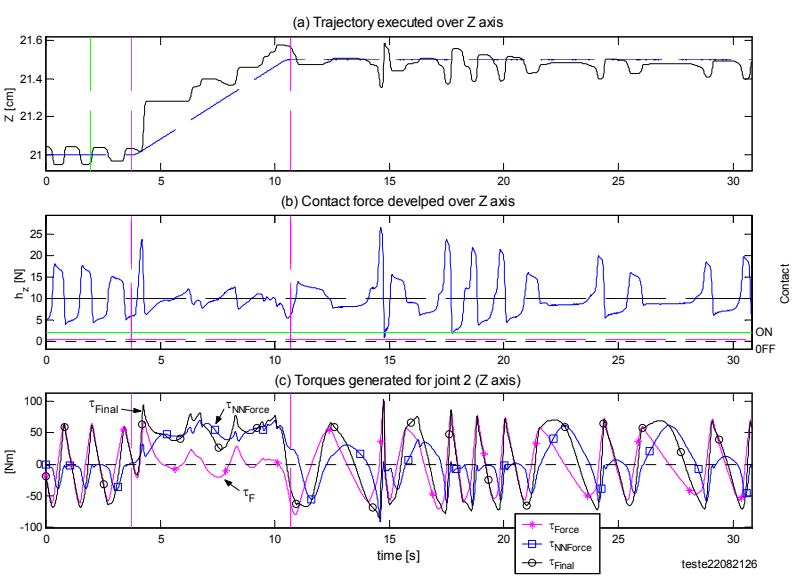

Figure 8. Results achieved by RNf-3. 

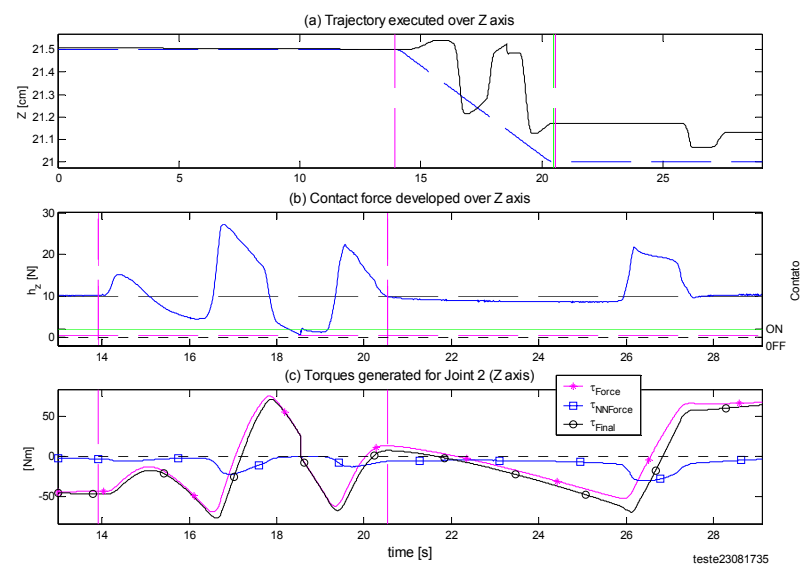

Figure 9. Results achieved by RNf-4..

\section{Conclusions}

This paper has presented an attempt to do force control using ANN performing in parallel with a conventional controller. The use of a conventional controller performing in parallel with the ANNs is advantageous to maintain the robustness of the system when the ANN become saturated (due to high learning rates) and is important to force the readjustment of the synaptic weights of the ANN used when occurs changes in the state of the robot-environment system. Only the integrate position controller performs very successfully. The other ANNs used in the force loop control could not capture the dynamic behavior of the system.

It would be realized the high computational resources required by the ANNs performing in parallel with a PI/PID controller. In fact, it was not possible to reduce the sampling time used by the hybrid integrate controller for $2(\mathrm{~ms})$ down to $1(\mathrm{~ms})$ - the quantity of floating point operations required have exhausted the resources of the actual CPU of Inter robot. As force control algorithm requires faster sampling times than positional control due more accelerated dynamic responses, probably the 2 (ms) sampling time used was not fast enough to allow the ANNs to capture the dynamics of the system robotenvironment. Maybe a promise solution could be the use of only one ANN which acts with the two controllers, one of the positional loop and the other of the force loop as showed in figure 10. In this case, this ANN will work with a mix of the resulting input data used before for each of the loop of the hybrid control structure and will produce the total torques needed by the robot after they pass the matrix selection (S) to separate the ones related to the position or the force control loop.

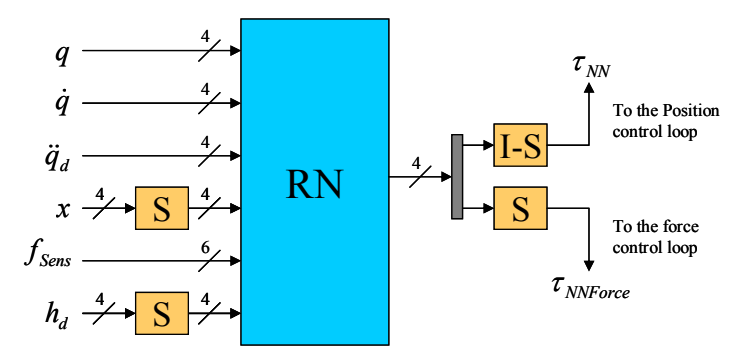

Figure 10. New proposal of using an ANN inside an hybrid force/control structure.

\section{References}

[1] Girosi, F. and Poggio, T., "Networks and the best approximation property", in M. M. Gupta and D. H. Rao (eds), Neuro-Control Systems, Theory and Applications, IEEE Pres: Piscataway, NJ, 1993., pp. 257-264.

[2] Haykin, S., Neural Networks: A Comprehensive Foundation, $2^{\text {nd }}$. Ed., Prentice Hall: New Jersey, 1999.

[3] Katic, D. and Vukobratovic, M., "Connectionist based robot control: an overview", $13^{\text {th }}$ IFAC, vol. 1b-05, 6, San Francisco, 1996, pp. 169-174.

[4] Kiguchi, K. and Fukuda, T., "Intelligent position/force controller for industrial robot manipulators - applications of fuzzy neural networks", IEEE Trans. On Idustrial Electronics, vol. 44, no. 6, 1997, pp. 753-761.

[5] Kim, Y. H., and Lewis, F. L., "Neural network output feedback control of robot manipulators", IEEE Trans. On Robotics and Automation, vol 15, no. 2, 1999, pp. 301309.

[6] Kuo, R. J.; “A robotic polishing system through fuzzy neural networks", Computers in Industry, Vol 32, 1997, pp. 273-280.

[7] Lightbody, G. and Irwin, G. W., "Nonlinear control sstrcutures based on embedded neural systems models", IEEE Trans. On Neural Networks, vol. 8, no. 3, 1997, pp. 553-567.

[8] Morris , A. S., and Khemanissia, S, "Artificial neural networks based intelligent robot dynamic control", in A. M. S. Zalazala and A. S. Morris (eds), Neural Networks for Robotic Control - Theory and Applications, chapter 2, Ellis Horwood: Great Briain, pp. 26-63.

[9] Narendra, K. S., "Neural networks for real-time control", $36^{\text {th }}$ IEEE Conference on Decision and Control, San Diego, CA, 1997, pp. 1026-1031.

[10] Sciavicco, L. and Siciliano, B., Modeling and Control of Robot Manipulators, McGraw-Hill, 1996.

[11] Volpe, R. A., "Real and Artificial Forces in the Control of Manipulators: Theory and Experiments", PhD Thesis, Dept. of Physics, Robotics Institute, Carnegie Mellon University, Pittsburgh, PA, 1990.

[12] Zeng, G. and Hemani, A. "An overview of robot force control", Robotica, Vol 15, 1997, pp. 473-482. 